\title{
Long term variability study for the radio galaxy M87 with MAGIC
}

\section{Priyadarshini Bangale $^{* a}$, Marina Manganaro ${ }^{b, c}$, Cornelia Schultz $^{d, e}$, Pierre Colin $^{a}$ and Daniel Mazin ${ }^{a, f}$ for the MAGIC Collaboration}

${ }^{a}$ Max-Planck-Institut für Physik, D-80805 München, Germany

${ }^{b}$ Inst. de Astrofísica de Canarias, E-38200 La Laguna, Tenerife, Spain

${ }^{c}$ Universidad de La Laguna, Dpto. Astrofísica, E-38206 La Laguna, Tenerife, Spain

${ }^{d}$ Università di Padova I-35131 Padova, Italy

${ }^{e}$ INFN, I-35131 Padova, Italy

${ }^{f}$ Japanese MAGIC Consortium, ICRR, The University of Tokyo, Japan

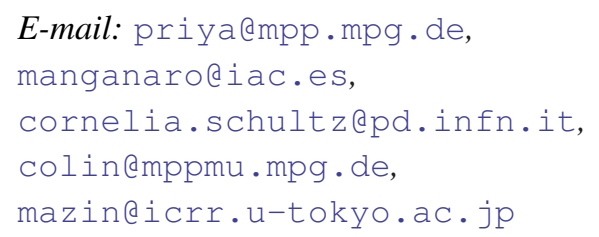

M87 is one of the closest known extragalactic very high energy (VHE, E > $100 \mathrm{GeV}$ ) object located in the Virgo cluster of galaxies at a distance of $16 \mathrm{Mpc}(z=0.00436)$. It is the first radio galaxy detected in the $\mathrm{TeV}$ regime, well studied from radio to $\mathrm{X}$-ray energies. The structure of its relativistic plasma jet, which is misaligned with respect to our line of sight, is spatially resolved in X-ray (Chandra), optical and radio (VLA/VLBA) observations. In 2005, gamma-ray emission at $\mathrm{TeV}$ energies was detected for the first time in M87. The VHE gamma-ray emission displays strong flux variability on timescales as short as a day. For more than 10 years, along with $\mathrm{X}$-ray, optical and radio bands, it has been monitored in the $\mathrm{TeV}$ band by imaging atmospheric Cherenkov telescopes MAGIC, H.E.S.S and VERITAS. In 2008 and 2010, M87 underwent several periods of $\mathrm{TeV}$ activities, and rapid flares with short timescale variability were detected. MAGIC continued to monitor M87 but no major flares were detected since 2010. However, the monitoring data set allows us to study the source in quiescent flux state. Here we present the status of these studies using the data from the last 4 years of MAGIC observations.

The 34th International Cosmic Ray Conference,

30 July- 6 August, 2015

The Hague, The Netherlands

\footnotetext{
* Speaker.
} 


\section{Introduction}

Messier 87, commonly known as M87, is a giant elliptical radio galaxy of Fanaroff-RileyI-type (FR I, [1]) in the Virgo Cluster, located at a distance of $16 \mathrm{Mpc}$ [2]. It is powered by a super-massive black hole of $(3.2 \pm 0.9) \times 10^{9} \mathrm{M}_{\odot}$ [3]. Its jet, which is misaligned with respect to our line of sight, originating from the core, extends to 20" ([4]; equivalent to $2 \mathrm{kpc}$ projected linear distance) and it was the first jet ever observed [5]. It is spatially resolved in X-ray (Chandra), optical and radio (VLA/VLBA) observations. M87 jet contains multiple features seen in radio, optical and X-ray termed as 'knots'. The closest feature to the nucleus is the knot 'HST-1', which is 0.86 " (70 pc, projected) away. At X-rays, Chandra has the angular resolution to separate the two components, showing a complex behaviour [6]. The angular resolution of gamma-ray observatories $\left(\sim 0.1^{\circ}\right)$ is too large to resolve the 20 " jet. However, the observed variability can be used to constrain the size of the emission region by requiring that the variability time scale is longer than the light travel time through the emission region. The measured day-scale variability at very high energy (VHE, $\mathrm{E}>100 \mathrm{GeV}$ ) implies a very compact source. The outer lobs are excluded as possible sites for VHE emission. Only close to the core or HST-1 are possible sites for this emission. Although this information alone is not enough to reveal the emission location, the correlation between the VHE emission and multiwavelength data in which the source is resolved provides a unique opportunity to localize the VHE process occurring in active galactic nuclei.

The first hint of VHE $\gamma$-ray emission reported by the High-Energy-Gamma-Ray Astronomy (HEGRA) collaboration [7], triggered extensive observations by the next generation Imaging Atmospheric Cherenkov telescopes (IACTs). The High Energy Stereoscopic System (H.E.S.S) collaboration firmly established M87 as an emitter above $730 \mathrm{GeV}$ and revealed flux variability on time scales of two days in 2005, suggesting the emission region of the $\gamma$-rays being very compact, with a dimension similar to the Schwarzschild radius of the central black hole [8].

The first reported detection of $\gamma$-ray emission from M87 by the Major Atmospheric GammaRay Imaging Cherenkov (MAGIC) happened in 2005, and results of those observation together with 2006 and 2007 data were reported in [9]. During the flare of 2008, MAGIC detected the source as well, observing a flux variability on time scales as short as a day [10]. For more than 10 years, along with X-ray, optical and radio bands, M87 has been monitored in the TeV band by MAGIC, H.E.S.S and VERITAS [14, 15]. According to the available VHE gamma-ray data there were in total three periods of high activity: 2005, 2008 and 2010. During these high TeV activities, rapid flares with short time scale variability were detected. Since the monitoring in VHE gamma rays is not very dense it cannot be excluded that more flaring periods took place in the last years. No major flares were detected since 2010, however, the monitoring data set allows us to study the source in quiescent flux state. In case of M87, most of the spectral modelling was done to interpret high or flaring states, whereas detailed characterisation of the source's lower emission levels is still lacking. Thus it is important to study the quiescent or low emission state as its origin of emission could be different from the flare, at least spread in much larger scale. Thus here we present the status of these studies using the data from the last 4 years of MAGIC observations. 


\section{Observations \& Analysis}

MAGIC is a stereoscopic system of two 17-m diameter IACTs situated at the Roque de los Muchachos, on the Canary island of La Palma $\left(28.75^{\circ} \mathrm{N}, 17.86^{\circ} \mathrm{W}\right)$ at a height of $2200 \mathrm{~m}$ above sea level. Since the end of 2009, it has been operating in stereoscopic mode with a trigger threshold of $\sim 50 \mathrm{GeV}$. During 2011 and 2012, MAGIC underwent a major upgrade in two stages. First, in summer 2011, the readout electronics of the telescopes were upgraded and in summer 2012, the camera of the MAGIC-I was replaced by a uniformly pixelized one, as the camera of MAGICII [16]. With the new system the integral sensitivity achieved is of $(0.66 \pm 0.03) \%$ of the Crab nebula flux above $220 \mathrm{GeV}$ in 50 hours at low zenith angles [17].

M87 observations were performed during December-July (visibility from La Palma) in each year from 2012 to 2015 at zenith angles ranging from $15^{\circ}-50^{\circ}$ during dark time and under Moon light conditions. The data were taken in the so-called wobble-mode [18] alternating the pointing direction between four sky positions at a $0.4^{\circ}$ offset from the source. To evaluate the residual background of the observation, three control regions with the same gamma-ray acceptance as the ON region were used to estimate the residual background recorded together with the signal. After the quality cuts, a total of $157 \mathrm{hrs}$ (Table 1) of effective observation time of good data were used for further analysis.

Data were analyzed using the standard MAGIC reconstruction software (MARS) [19]. The recorded shower images were calibrated, cleaned and parametrized according to [21] for each telescope individually. Since the Moon light increases the background signal in each pixel, data were processed by applying an image cleaning higher than the standard one to the data. The cleaning levels were optimized based on the percentage of pedestal events (artificial triggered events which includes electronic noise and night sky background (NSB), without any showers) surviving the cleaning. Monte Carlo simulations were tuned to the Moon light conditions by increasing the fluctuations of pedestal baseline to mimic the effect of a higher NSB level. For the reconstruction of the shower arrival direction the random forest regression method [22] with the implementation of stereoscopic parameters [23] was used. The $\gamma$-hadron separation was performed using the random forest method [24], which is based on both individual image parameters from each telescope and stereoscopic information such as the shower impact point and the shower height maximum. Energy look-up tables were used for the energy reconstruction. Further details on the stereo MAGIC analysis can be found in [25].

\section{Results}

MAGIC detected M87 in every yearly campaign between 2012 and 2015. Table 1 lists the effective observation time and significance of the VHE $\gamma$-ray signal observed from M87 between 2012 and 2015. The significance of the detection was calculated according to Li\&Ma eq. 17 [26].

The daily- and monthly-binned light curves above $300 \mathrm{GeV}$ are shown in Figure 1 and the mean integral flux of each year, which was obtained by a constant fit to the monthly-binned light curves, are reported in Table 1. Variability on different times scales was investigated. No significant variability observed on yearly and monthly scales because the flux averages out with the days without any signal. Hint of variability on daily scale observed in 2013 (probability for fit with 


\begin{tabular}{c|c|c|c}
\hline \hline Year & $\mathrm{T}_{\text {eff }}[\mathrm{h}]$ & Significance $[\sigma]$ & $F_{E>300 \mathrm{GeV}}\left[\times 10^{-12} \mathrm{~cm}^{-2} \mathrm{~s}^{-1}\right]$ \\
\hline 2012 & 38.75 & 5.4 & $1.15 \pm 0.35$ \\
2013 & 34.82 & 8.7 & $1.87 \pm 0.30$ \\
2014 & 49.88 & 7.2 & $1.48 \pm 0.22$ \\
2015 & 32.72 & 5.9 & $1.25 \pm 0.33$ \\
\hline \hline
\end{tabular}

Table 1: Effective observation time, significance and mean integral flux ( $E>300 \mathrm{GeV})$ of the VHE $\gamma$-ray signal observed from M87 between 2012 and 2015. The mean integral flux obtained from a constant fit to the monthly binned light curves (Figure 1).
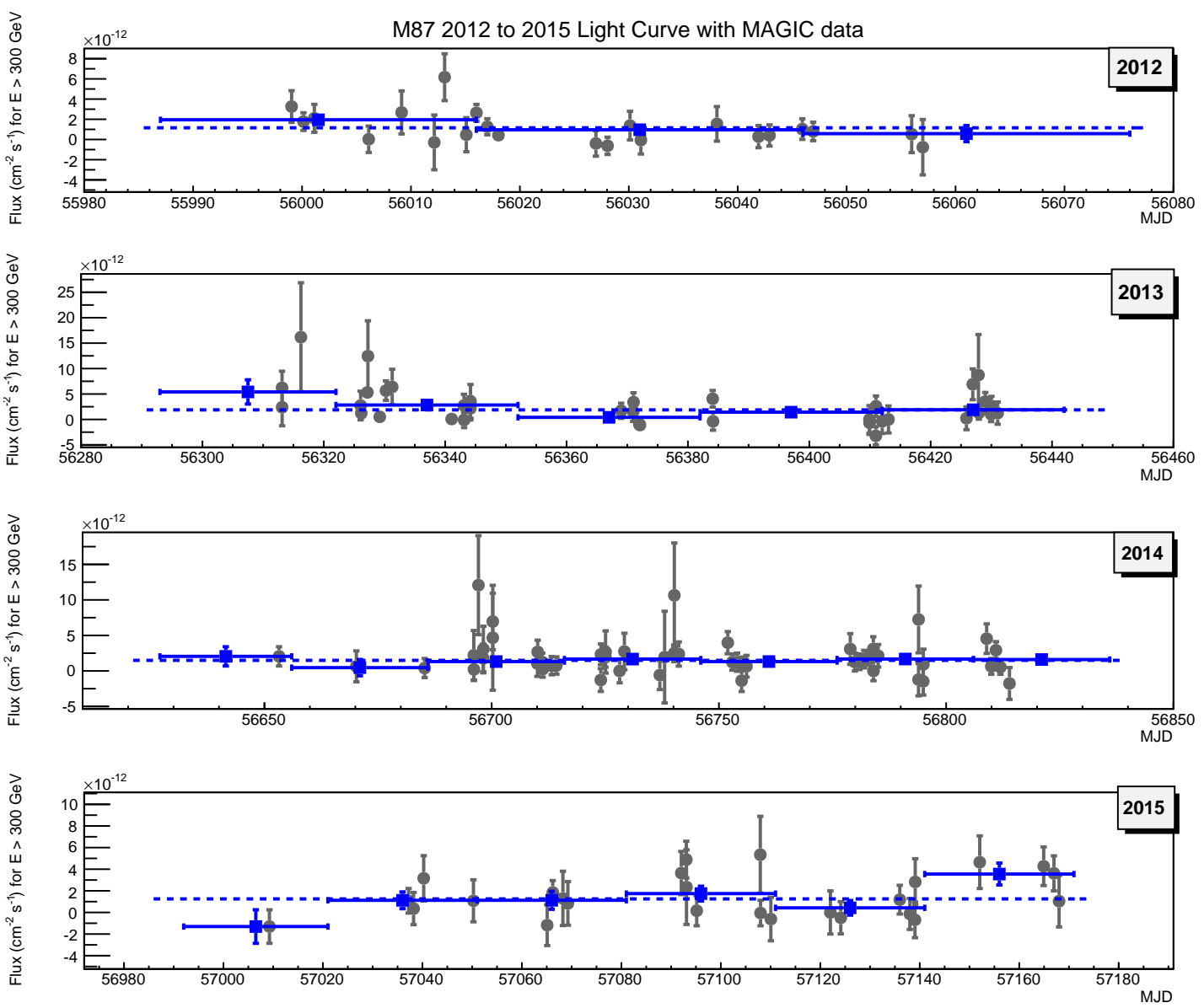

Figure 1: Daily- (gray) and monthly- (blue) binned light curves from 2012 to 2015 (top to bottom). The mean flux from a fit with constant to the monthly-binned light curves is indicated by a blue dotted line (reported in Table 1).

constant of $0.3 \%$ ), while for other years compatible with a constant flux (probability for fit with constant > 38\%). For 2013 data, assuming an additional systematic uncertainty of $11 \%$ of the measured flux [17] we obtain probability for fit with constant of $0.9 \%$, which still shows hint of variability on daily scale. 
We calculate an integral flux $\mathrm{F}(E>300 \mathrm{GeV})$ for each data set using the energy spectra and integral fluxes information on the previous data from the literature (see [9], [13]), shown in (Table 2). The integral flux level above $300 \mathrm{GeV}$ between 2012 and 2015 is the lowest observed since 2005.

\begin{tabular}{c|c} 
Year & $F_{E>300 \mathrm{GeV}}\left[\mathrm{cm}^{-2} \mathrm{~s}^{-1}\right]$ \\
\hline \hline $2005-2007$ & $(1.88 \pm 0.44) \times 10^{-12}$ \\
\hline 2008 low state & $(11.0 \pm 4.4) \times 10^{-12}$ \\
\hline 2008 high state & $(16.8 \pm 3.2) \times 10^{-12}$ \\
\hline $2012-2015$ & $(1.60 \pm 0.18) \times 10^{-12}$
\end{tabular}

Table 2: Comparison of the integral fluxes above $300 \mathrm{GeV}$ observed between 2005 and 2007 [9], in 2008 [13] and between 2012 and 2015 during both low and high states of the source. The integral fluxes have been extrapolated from the simple power-law fits to the observed spectra.

\section{Conclusions}

MAGIC continues to monitor M87 ( $\sim 40$ hrs per year) and detected the source in every yearly campaign between 2012 and 2015. No flare was detected and no clear variability was observed in 2012, 2014 and 2015 data on daily and monthly time scales. A hint for variability ( $3 \sigma$ level) was found in 2013 data on a daily time scale. The hint of the variability remains at a similar significance level even when variable systematic uncertainties of the MAGIC measurements are taken into account. The VHE $\gamma$-ray flux level above $300 \mathrm{GeV}$ between 2012 and 2015 is the lowest observed since 2005 .

\section{Acknowledgments}

We would like to thank the Instituto de Astrofísica de Canarias for the excellent working conditions at the Observatorio del Roque de los Muchachos in La Palma. The financial support of the German BMBF and MPG, the Italian INFN and INAF, the Swiss National Fund SNF, the ERDF under the Spanish MINECO (FPA2012-39502), and the Japanese JSPS and MEXT is gratefully acknowledged. This work was also supported by the Centro de Excelencia Severo Ochoa SEV-2012-0234, CPAN CSD2007-00042, and MultiDark CSD2009-00064 projects of the Spanish Consolider-Ingenio 2010 programme, by grant 268740 of the Academy of Finland, by the Croatian Science Foundation (HrZZ) Project 09/176 and the University of Rijeka Project 13.12.1.3.02, by the DFG Collaborative Research Centers SFB823/C4 and SFB876/C3, and by the Polish MNiSzW grant 745/N-H.E.S.S-MAGIC/2010/0. 


\section{References}

[1] B. L. Fanaroff, J. M. Riley, The morphology of extragalactic radio sources of high and low luminosity, MNRAS, 161 (1974), 31-36

[2] L. M. Macri, et al., The Extragalactic Distance Scale Key Project. XVIII. The Discovery of Cepheids and a New Distance to NGC 4535 Using the Hubble Space Telescope, ApJ, 521 (1999), 155

[3] F. Macchetto, A. Marconi and D.J. Axon, The supermassive black hole of M87 and the kinematics of its associated gaseous disk, The Astrophysical Journal, 489 (1997), 579, [arXiv:9706252]

[4] H. L. Marshall, et al.,A High-Resolution X-Ray Image of the Jet in M87, The Astrophysical Journal, 564 (2002), 683, [arXiv:0109160]

[5] H. D. Curtis, Descriptions of 762 Nebulae and Clusters Photographed with the Crossley Reflector, Publications of the Lick Observatory (University of California Press), 13 (1918), 31

[6] W. E. Harris, The Globular Cluster System in M87: A Wide-Field Study with CFHT/Megacam, The Astrophysical Journal, 703 (2009), 939, [arXiv:0908.1120]

[7] F. Aharonian, Is the giant radio galaxy M 87 a TeV gamma-ray emitter?, Astronomy and Astrophysics, 403 (2003), L1, [arXiv:0302155]

[8] F. Aharonian, Fast Variability of Tera-Electron Volt $\gamma$ Rays from the Radio Galaxy M87, Science, 314 (2006), 1424, [arXiv:0612016]

[9] J. Aleksić, et al., MAGIC observations of the giant radio galaxy M87 in a low-emission state between 2005 and 2007 ,Astronomy and Astrophysics, 544 (2012), A96, [arXiv:1207.2147]

[10] V. A. Acciari,Observation of gamma-ray emission from the galaxy M87 above $250 \mathrm{GeV}$ with VERITAS, The Astrophysical Journal, 679 (2008), 397 [arXiv:0802.1951]

[11] V. A. Acciari, VERITAS 2008 - 2009 monitoring of the variable gamma-ray source M87, The Astrophysical Journal, 716 (2010), 819, [arXiv:1005.0367]

[12] A. A. Abdo,Fermi/Large Area Telescope Bright Gamma-Ray Source List, The Astrophysical Journal Supplement, 183 (2008), 46 [arXiv:0902.1340]

[13] J. Albert, et al., Very High Energy Gamma-Ray Observations of a Strong Flaring Activity in M87 in 2008 February,Astrophysical Journal, 685 (2008), L23, [arXiv:0806.0988]

[14] V. A. Acciari,Radio Imaging of the Very-High-Energy Gamma-Ray Emission Region in the Central Engine of a Radio Galaxy - VERITAS and H.E.S.S and MAGIC Collaborations, Science, 325 (2009), 444 [arXiv:0908.0511]

[15] A. Abramowski, et al., The 2010 very high energy gamma-ray flare \& 10 years of multi-wavelength observations of M 87, The Astrophysical Journal, 746 (2012), 151

[16] J. Aleksić, et al., The major upgrade of the MAGIC telescopes, Part I: The hardware improvements and the commissioning of the system, Astroparticle Physics, (2015) [arXiv:1409.6073]

[17] J. Aleksić, et al.,The major upgrade of the MAGIC telescopes, Part II: The achieved physics performance using the Crab Nebula observations, (2014), [arXiv:1409.5594]

[18] V. P. Fomin, et al., New methods of atmospheric Cherenkov imaging for gamma-ray astronomy. I. The false source method, APh, 2 (1994), 137

[19] R. Zanin, et al., MARS, the MAGIC analysis and reconstruction software, Proc of the 33rd ICRC, Rio de Janeiro, 2013, 2, 773 
[20] E. Aliu, et al., Improving the performance of the single-dish Cherenkov telescope MAGIC through the use of signal timing, APh, 30 (2009), 293, [arXiv:0810.3568]

[21] A. M. Hillas, Cerenkov light images of EAS produced by primary gamma, Proceedings of the 19th ICRC, La Jolla, 3 (1985), 445

[22] J. Aleksić, et al., Search for an extended VHE gamma-ray emission from Mrk 421 and Mrk 501 with the MAGIC Telescope, Astronomy and Astrophysics, 524 (2010), A77, [arXiv:1004.1093]

[23] S. Lombardi, et al., Advanced stereoscopic gamma-ray shower analysis with the MAGIC telescopes, Proceedings of the 32nd ICRC, Beijing, 3 (2011), 262, [arXiv:1109.6195]

[24] J. Albert, et al., Implementation of the Random Forest method for the Imaging Atmospheric Cherenkov Telescope MAGIC,Nuclear Instruments and Methods in Physics Research, 588 (2008), 424, [arXiv:0709.3719]

[25] J. Aleksić, et al., Performance of the MAGIC stereo system obtained with Crab Nebula data, Astroparticle Physics,35 (2012), 435, [arxiv:1108.1477]

[26] T. Li and Y. Ma, Analysis methods for results in gamma-ray astronomy,ApJ, 272 (1983), 317 